\title{
Physiology and Psychology of Dreams
}

\author{
Alan S. Eiser, Ph.D. ${ }^{1}$
}

\section{ABSTRACT}

The discovery of the close association between rapid eye movement (REM) sleep and dreaming and development of sleep laboratory techniques ushered in a new era in the study of dreams. For the first time, direct and systematic investigation could be made of such topics as the occurrence, qualities, recollection, and childhood development of dreaming. Experimental methodologies permitted investigation of the responsiveness of dreams to external stimulation and the effects of deprivation of REM sleep. Much effort was devoted to searching for parallels between physiological aspects of REM sleep and characteristics of associated dreams, with modest results. The leading theory of dreaming in the early decades of this research was the psychoanalytic, which views dreams as highly meaningful reflections of unconscious mental functioning. With developments in understanding of the neurophysiology of REM sleep, new theories of dreaming were proposed. The most prominent, the activation-synthesis hypothesis, derived its view of dreaming directly from the neurophysiology of REM sleep, in particular the role of the brain stem, and in its original form regarded dreams as not essentially meaningful. Further developments in neurobiological research, including lesion and brain imaging studies, have established a clearer view of the functional neuroanatomy of REM sleep and dreaming. To what degree, and in what way, implications can be drawn from these findings for the psychology of dreaming is controversial. Some more recent theories of dreaming emphasize an adaptive function related to emotion and a role in learning and memory consolidation.

KEYWORDS: Dreams, REM sleep, psychology, neurophysiology, psychoanalysis, activation-synthesis

Objectives: On completion of this article, the reader will be able to develop a better understanding of the neurophysiology of dreaming, and the controversies about the psychology of dreams.

Accreditation: The Indiana University School of Medicine is accredited by the Accreditation Council for Continuing Medical Education to provide continuing medical education for physicians.

Credit: The Indiana University School of Medicine designates this educational activity for a maximum of 1 Category 1 credit toward the AMA Physicians Recognition Award. Each physician should claim only those hours of credit that he/she actually spent in the educational activity.

Disclosure: Statement has been obtained regarding the author's relationships with financial supporters of this activity, use of trade names, investigational products, and unlabeled uses that are discussed in the article. The author has nothing to disclose.

The discovery of rapid eye movement (REM) sleep and its close association with dreaming, ${ }^{1}$ and the subsequent elaboration of the non-REM (NREM)/ REM sleep cycle, ${ }^{2}$ ushered in a new era in the study of dreams. The link between dreaming and a specific, universal, and regularly occurring stage of sleep permitted direct and systematic investigation of a range of questions and topics that had previously remained in the

Sleep in Neurological Practice; Editor in Chief, Karen L. Roos, M.D.; Guest Editor, Alon Y. Avidan, M.D., M.P.H. Seminars in Neurology, Volume 25, Number 1, 2005. Address for correspondence and reprint requests: Alan S. Eiser, Ph.D., Lecturer, Department of Neurology, University of Michigan Sleep Disorders Center, UH-8D8702, 1500 East Medical Center Drive, Ann Arbor, MI 48109-0117. ${ }^{1}$ Departments of Neurology and Psychiatry, University of Michigan Sleep Disorders Center, Ann Arbor, Michigan. Copyright (C) 2005 by Thieme Medical Publishers, Inc., 333 Seventh Avenue, New York, NY 10001, USA. Tel: +1(212) 584-4662. 0271-8235,p;2005,25,01,097,105,ftx,en;sin00348x. 
realm of theory and speculation. New experimental approaches to dreaming were developed and tried. Indeed, the interest generated in studying dreams with the new technology was perhaps the major impetus in the first two decades of sleep laboratory research. In turn, as the science of sleep and the field of sleep disorders have subsequently advanced, new findings, many in the realm of neurobiology, and new technologies, including those in brain imaging, have been applied to the study of dreaming. Efforts to approach an understanding of dreams on the basis of REM sleep neurophysiology have been made. The degree to which these new models have supplanted earlier, psychologically based theories of dreaming remains controversial.

\section{DREAMS AND THE SLEEP CYCLE}

In their earliest report, Aserinsky and Kleitman ${ }^{1}$ found that $74 \%$ of awakenings from REM sleep resulted in recall of a dream, as compared with only $9 \%$ of awakenings from NREM sleep. The association between dreaming and REM sleep was subsequently replicated by many other investigators; typically, around $80 \%$ of REM awakenings yield dreams. The much greater likelihood of obtaining a dream report from REM sleep led some early researchers to conclude that all dreaming, and even all mental activity, during sleep occurred in REM sleep, with reports from NREM sleep reflecting recall of mental activity from an earlier REM period, or mentation arising during the arousal itself. On the other hand, several laboratories reported much higher frequencies of NREM reports than in the earliest studies. ${ }^{3}$ Some of the differences reflected less stringent criteria for what constitutes a dream. It became clear over time that there is a good deal of mental activity that occurs during NREM sleep. Typically, it is more thoughtlike, fragmentary, and related to daily concerns than the vivid, hallucinatory, predominantly visual narratives that are most commonly reported from REM sleep. But even this distinction appears not to be absolute. There is now wide acceptance of the view that some dreaming that is indistinguishable from REM sleep dreaming occurs in NREM sleep, most frequently in the sleep-onset period.

Notwithstanding these exceptions in the relationship between REM sleep and dreaming, the association is close enough that it became possible with the new research to establish approximate answers to such questions as the frequency, length, and time of occurrence of dreaming. The four or five REM periods that people typically have in a night can be taken as a rough estimate of the number of dreams per night. These REM periods, and associated dreaming, occupy $\sim 20 \%$ of the night in young adults. REM periods, and hence most dreams, occur in a 90-minute cycle, with a period of NREM sleep followed by REM sleep. Individual REM periods may last from a few minutes to over an hour, with REM periods becoming longer the later it is in the night. There is evidence the dream is coextensive with the REM period and that the time length of dreams, as subjectively experienced by the dreamer and objectively rated by experimenters, is closely related to the real-time length of the corresponding REM period. ${ }^{4}$ This indicates that dreams are longer later in the night and most concentrated in the last third of the night, with the exception of sleep-onset dreaming. The typical content of dreams has been studied and frequently reported to be more mundane and more closely linked to daytime concerns than is popularly thought. ${ }^{5}$ The suggestion has been made that this difference is due to the fact that the dreams that tend to be recalled outside the laboratory are those that are more vivid and bizarre and therefore may not be representative. On the other hand, the question has been raised whether dreams in a laboratory situation, where the dreamer knows he will be awakened and his dream elicited, may be less "dreamlike" than those at home. Another reported finding is that dysphoric affects, especially fear and anxiety, predominate in dreams. ${ }^{5}$

Whether or not a dream is recalled at all depends a great deal on the timing of awakening. Awakenings while a REM period is ongoing most often result in dream recall; recall rate drops off rapidly if the awakening is delayed until after the REM period has ended. Findings have been interpreted to suggest that most dreams are lost to recall by 8 minutes, ${ }^{4}$ or even within seconds, ${ }^{6}$ after the end of the REM period. It appears that the majority of dreams are "forgotten" only in the sense that they have never been committed to memory.

Early attempts were made to look at the sequence of dreams across a night. Some preliminary results suggested that the dreams of a given night may all deal with the same emotional conflict, or a limited number of conflicts. ${ }^{7}$ It was also reported that themes and images may be repetitively present, in varying contexts, in mentation from NREM awakenings across a night and that elements in NREM mentation may also be seen in dream reports from REM sleep on the same night. When this occurs, the dreams "emerge as the most vivid and memorable part of a larger fabric of interwoven mental activity during sleep." 8

Other physiological features of REM sleep have been considered in terms of their relevance to dreaming. One is the muscle paralysis that accompanies that state, viewed as protection against acting out dreams. The rapid eye movements that characterize this sleep stage have been thought by some investigators to stem from the eyes following the hallucinated imagery of the dream. Some dramatic instances in which this view, known as the "scanning hypothesis," appears to be confirmed have been reported, ${ }^{4,9}$ but how generally this is the case remains a matter of controversy. Finally, 
the cycle of penile erection in males and vaginal blood engorgement in females that occurs in close temporal association to the periods of REM sleep has been suggested to be an indication of a state of drive activation linked to dreaming.

\section{CHILDHOOD DEVELOPMENT OF DREAMING}

Some attention has been paid to developmental aspects of dreaming. The amount of REM sleep (or active sleep, its developmental precursor) is at its maximum at birth, comprising $50 \%$ of sleep time, and decreases to the adult level of 20 to $25 \%$ by 3 to 5 years of age. However, it is very difficult to know what these facts mean for dreaming; the younger the child, the more limitations in linguistic and conceptual abilities, and in memory capacity, create obstacles to investigating the child's experience during REM sleep. Laboratory studies have provided evidence that dream recall is possible from REM sleep awakenings as early as 2 years of age. ${ }^{10}$ However, reports of dreams are obtained much less frequently the younger the age of the child. Foulkes, who has done the most extensive work in this area, reported that a median figure of only $15 \%$ of REM sleep awakenings in children 3 to 5 years of age yielded dreams. ${ }^{11} \mathrm{He}$ described the dreams of young children as brief and static; barnyard animals were the most frequent characters, and body states were often represented. He reported a gradual development through the 7- to 9-year-old age range in recall frequency and key dream qualities to something more like what is familiar from adults. ${ }^{11}$ Questions have been raised regarding how accurately the laboratory-collected dreams reflect dreaming experience in very young children, given differences from the impressions obtained from parents who are much closer to the children and in clinical populations, ${ }^{12,13}$ but it is a centrally important observation that dreaming undergoes a process of development and that it must be viewed in the context of the child's developing cognitive capacities.

\section{EXPERIMENTAL MANIPULATIONS}

A variety of experimental manipulations have been tried in an effort to better understand the nature and process of dreaming. For example, subjects have been exposed to different kinds of stimulation during or before sleep and the effects on dreaming noted. It has been found that stimuli given during REM sleep may be incorporated in dreams, either in a direct or in a transformed way. A very early study of this kind, by Dement and Wolpert, ${ }^{14}$ found that the frequency with which subjects incorporate different kinds of stimuli given during REM sleep varies with the type of stimulus. A spray of water on the skin was the most frequently incorporated stimulus, at a frequency of $42 \%$. An example of a direct incorporation was in a dream of being squirted by someone; an indirect incorporation was seen in a dream of a leaky roof.

A very extensively explored experimental manipulation, pioneered by Dement, is the deprivation of REM sleep, often referred to as dream deprivation in the early days of sleep research when dreaming was thought to occur exclusively in REM sleep. It was consistently found that a subject deprived of REM sleep would make more and more frequent attempts to enter that stage; after the period of REM deprivation was terminated, the subject would show a compensatory increase in REM time, called REM rebound. ${ }^{15,16}$ This was taken as evidence that REM sleep, and dreaming, must perform a vital function. An early hypothesis, based in part on psychoanalytic thought, was that subjects deprived of dreaming would become psychotic, and initial findings ${ }^{17}$ were seen as congruent with this expectation. However, this view was not borne out by later work done with better experimental methodology ${ }^{18}$; there was even a study by Vogel and colleagues that showed clinical improvement with prolonged REM sleep deprivation in patients with endogenous depression. ${ }^{19}$

A variety of studies in cats, rats, and mice provided evidence that prolonged REM deprivation may lead to hypersexual, hyperaggressive, and increased intracranial self-stimulation behavior, which has been interpreted as supporting the view that dreaming is linked to basic drives and pleasure-seeking. ${ }^{20-22}$ Also investigated extensively has been the view that REM sleep is important for learning and memory consolidation and that these functions in turn are impaired by REM deprivation. Early studies yielded mixed results; more recent efforts, and conceptual refinements, may result in clearer evidence of such relationships, ${ }^{23,24}$ but this remains an area of considerable controversy. ${ }^{25}$

\section{DREAM DISORDERS}

Another important yield of laboratory research has been an enhanced understanding of clinical phenomena related to dreaming. The distinction between nightmares and night terrors has been elucidated. ${ }^{26}$ The former are now usually understood as elaborate REM dreams that end in anxiety leading to awakening, with modest autonomic arousal and typically rapid alerting with detailed recall. Night terrors, on the other hand, generally are arousals following brief, fragmentary imagery typically in stage 3 or 4 sleep, with very intense autonomic arousal, delay in becoming fully oriented and alert, and little or no recall. Sleepwalking, previously thought on clinical grounds to be associated with dreaming, typically arises out of NREM sleep in the absence of dreaming. ${ }^{27,28}$ On the other hand, a relatively recently described disorder called REM behavior disorder occurs in patients with defective muscle paralysis 
during REM sleep and involves the acting out of dreams. ${ }^{29}$

\section{PSYCHOPHYSIOLOGICAL PARALLELISM}

In addition to establishing basic knowledge about dreams, there has been a great deal of effort to capitalize on the REM sleep/dreaming connection as a means of studying in more detail psychophysiological parallelism. The question has been posed, in a variety of ways, whether specific physiological characteristics of REM sleep would correlate with specific features and qualities in associated dreams. Early studies reported connections between REM periods with denser eye movements and dreams that are more active with self-participation, ${ }^{14}$ vivid, and emotional. Some of these results were later called into question, as more sophisticated methodologies and analyses were employed. With respect to other physiological variables, better correlations tend to be obtained between periods of rapid physiological change and dream content. A notable example is the report by Fisher. ${ }^{30}$ Studying the cycle of penile erection roughly coincident with REM sleep in men, Fisher found that he was most successfully able to relate dream content to this physiological variable when the variable underwent a sudden sharp change. Periods of rapid detumescence correlated with dream content reflecting marked anxiety related to the appearance of aggressive threats toward the dreamer, or by the dreamer toward others, and rapid tumescence correlated with the appearance of overt or symbolic sexual content in the dream.

Later efforts to correlate dream content just before awakening with physiological characteristics in the same brief preawakening period drew upon the distinction between the tonic, or relatively unchanging, features of REM sleep (e.g., the low-voltage, mixedfrequency electroencephalogram and the muscle atonia) and the phasic events, or aspects occurring in discrete, relatively brief bursts, such as the rapid eye movements, muscle twitches, and respiratory irregularities. It was thought that phasic activity would be linked to qualities in dreams that make them "dreamlike." This work has been reviewed and summarized by Pivik. ${ }^{31}$ One relationship that was reported was between bursts of eye movements in REM sleep and the quality of "primary visual experience" (i.e., visual imagery simply seen without cognitive elaboration) in the associated dream content; "secondary cognitive elaboration" was more frequent during periods of REM sleep without eye movements or during NREM sleep. ${ }^{32}$ In general, however, associations that have been reported between phasic activity and dream characteristics have tended to be relatively weak. The same has been true when the concept of phasic activity has been extended to NREM sleep and its mentation. Perhaps with hindsight it is not surprising that strong correspondences between single physiological measures and ongoing, complex mental processes are difficult to obtain.

\section{PSYCHOLOGY OF DREAMING}

\section{Psychoanalytic Model}

During the early decades of sleep laboratory research, the most prominent extant theory of dreaming was the psychoanalytic, initially developed by Freud. ${ }^{33}$ Because this model has been quite influential in sleep research and continues to have strong adherents at the present time, it will be summarized in some detail. For Freud, the dream is a highly meaningful mental product that is the outcome of particular mental processes under the circumstances of sleep. The meaning of the dream is not apparent in the "manifest content" - the dream as recalled by the dreamer-but is to be found in the "latent dream thoughts," some of which are unconscious; the latent thoughts have to be uncovered by processes of association to the manifest elements of the dream. The latent content includes conflicted instinctual (i.e., sexual or aggressive) wishes originating in childhood, which are the motive force for the dream; the dream is an attempt at fulfillment of these wishes. At the same time, the dream contains "day residues," thought processes typically from the previous day, which are the point of contact between the unconscious wishes and current concerns. The latent dream thoughts are transformed into the manifest dream by what are called primaryprocess mechanisms, a primitive form of thinking predominant in dreams. Primary process mechanisms include condensation, the combining or fusing of two or more elements into a single one, and displacement, a shifting of emphasis from one element to a different one. Processes of symbolization are also involved in the transformation of latent thoughts into the manifest dream. The primary process mechanisms also subserve the need for disguise, or censorship, of the conflicted wishes, so that they will not arouse excessive anxiety and disturb sleep; censorship is diminished but not absent during sleep. Blocked from motor output due to the state of sleep, the mental activity undergoes a regressive process that results in the dream being represented perceptually, as a hallucination.

The psychoanalytic model was derived from the in-depth study of individual dreams in a context of intensive, richly detailed personal exploration. Certain aspects of the model clearly required revision in light of initial basic sleep laboratory findings. Hence, it is not just the state of sleep in general but rather REM sleep that is particularly facilitative of dreaming, and the occurrence of dreaming is largely preprogrammed, rather than based on the seemingly more happenstance circumstance of 
whether an instinctual wish is aroused during sleep. What has proven difficult is to devise truly adequate experimental tests of more central elements of the model. This has led some ${ }^{34}$ to question the scientific status of certain aspects, but the difficulty may reflect the very complexity that gives the model its value, the fact that it deals with covert or unconscious elements and that some of its postulates ("wish-fulfilling") concern basic assumptions about mental functioning not readily testable in the narrow arena of dreaming. A notable exception to the difficulty in devising appropriate tests is the experimental approach involving subliminal stimulation used by Shevrin and Fisher. ${ }^{35}$ A special subliminal stimulus previously demonstrated to evoke both primary- and secondary-process levels of responses was given prior to sleep. In free associations elicited after awakenings from REM and NREM sleep, it was demonstrated that primary-process associates to the stimulus predominated after REM sleep and secondary-process associates after NREM sleep, consistent with the view of a different level of cognitive processing in dreaming. The use of subliminal stimulation provided a means for tracking unconscious influences on dreams.

There is a wide spectrum of views in the field of sleep research regarding the status of the psychoanalytic theory of dreaming in contemporary thought, ranging from those who essentially completely repudiate the theory, regarding it as incompatible with findings in neurobiology $^{36}$ or in laboratory-collected dreams, ${ }^{11}$ to those who feel research findings are strikingly supportive of the most essential tenets of the model. ${ }^{37}$ For many, the explanatory richness and clinical utility of the theory give it continued importance in efforts to understand the psychology of dreaming. More recent developments in psychoanalytic theory, in the understanding of conflict, object relations (internal relatedness to people), and narcissism (development of the self and self-esteem), can in turn supplement the original emphasis on instinctual drives in providing further explanatory tools for understanding dreams.

As knowledge accumulated, more comprehensive efforts were made to link sleep laboratory discoveries to an overall psychology of dreaming. An early, monumental effort was that of Fisher, who provided a comprehensive summary of sleep laboratory findings up to 1965 and an in-depth, probing integration of them with psychoanalytic theory. ${ }^{38}$

\section{Activation-Synthesis Model}

As understanding of the neurobiology of REM sleep developed, efforts were made to utilize this knowledge as the basis for an approach to dreaming. Hobson and associates have been major contributors to the understanding of the sites and neurochemical interactions in the brain stem that are involved in the generation of
REM sleep. ${ }^{39,40}$ Out of this work, they developed a very different approach to dreaming, called the activationsynthesis hypothesis. ${ }^{40} \mathrm{~A}$ radical departure from previous views, this theory attempts to derive an explanation of dreaming by direct interpretation of the neurophysiology of REM sleep, based on what the authors call mind-body isomorphism - an assumption of an "identity of form" between physiological and psychological events. The model in its original form leans heavily on the fact of the generation of REM sleep in the pontine brain stem. In the activation portion of the model, the authors view the brain stem as providing random, direct stimulation of the forebrain, for example of the oculomotor, vestibular, and motor systems, accounting for the prominence of visual and movement elements in dreams. The forebrain is seen as attempting to synthesize the information that has been generated, perhaps by a process in which "best fits to the relative inchoate and incomplete data ... are called up from memory, the access to which is facilitated during dreaming sleep. ... The forebrain may be making the best of a bad job in producing even partially coherent dream imagery from the relatively noisy signals sent up to it by the brainstem." 40 The formal qualities of dreams are directly derived in this model from the properties of the brainstem stimulation. Dreams in this view are not essentially meaningful, though some meaning may accrue secondarily in the forebrain's efforts to make sense of its physiologically determined stimulation.

The activation-synthesis model has been widely influential in the field of sleep research and sleep disorders, becoming in many quarters the leading perspective on dreaming. At the same time, it has evoked great controversy and has been trenchantly criticized from the time of its inception to the present, most frequently for what is viewed as the arbitrary quality of its inferences from the neurophysiology and the failure to deal with NREM dreaming. ${ }^{41-43}$ The model has gradually evolved to allow for a greater and more functional role for the forebrain, particularly in the consolidation of learning and memory. Its most recent form, the AIM model, ${ }^{44}$ utilizes a three-dimensional space concept for explaining in neurobiological terms the entire spectrum of brainmind states on the basis of the variables of activation (level of brain activation), input source (external sensory versus internally generated), and modulation (neuromodulatory balance between aminergic and cholinergic influence, thought to affect mode of cognitive functioning). REM sleep, when most dreaming takes place, is thus seen as comprised of a high level of activation (hence the intense mental activity), internal rather than external input, and aminergic demodulation (related to the diminution of such qualities as logic, orientational stability, self-reflective awareness, and memory). Some of the Hobson group's current positions, suggesting a view of dreams as entailing an 
emotion-driven processing and consolidation of memory, seem closer to theories attributing significant meaning to dreams than their earlier views.

\section{LESION AND IMAGING STUDIES}

\section{Lesion Studies}

A major contribution to the understanding of dreaming and the brain has been provided by Solms. ${ }^{45-47}$ His approach has been to study neurological patients with lesions in a wide range of brain sites and correlate reported changes in recalled dreams with location of lesion and associated clinical syndrome. Interestingly, this clinicoanatomical approach, which has brought an entirely new area of data to the study of brain/dreaming relationships, is not in its essence dependent on considerations of stage of sleep or the technology of the sleep laboratory. Solms collected data from 332 neurological patients and combined his findings with widely scattered reports collated from the previous literature to develop a neuropsychology of dreaming. He found that lesions in two areas of the brain resulted in complete loss of dreaming. One area is at or near the parietal-temporooccipital (PTO) junction; both unilateral and bilateral lesions in this area lead to cessation of dreaming. Solms infers that the right parietal-temporo-occipital area is essential to dreaming for its function of spatial thought, and the left parietal-temporo-occipital area is required for quasi-spatial (symbolic) thought. The second type of lesion that leads to loss of dreaming is bilateral deep frontal lesions. Solms believes that this area contributes appetitive interest in the world, as well as regulatory influences necessary for elaborated volitional interest, and that these functions are essential for dreaming as well.

Solms reports additional findings of considerable interest. Patients with lesions in the visual association cortex experience loss of visual imagery in dreams, as well as loss of the capacity to conjure up visual imagery when awake; visual imagery in dreams and wakefulness is preserved with lesions in the primary visual cortex. The findings are similar in other sensory modalities. This suggests that dream imagery is "actively constructed through complex cognitive processes." ${ }^{.77}$ Lesions in various anterior limbic structures led to an inability to distinguish dreams from reality, often in conjunction with increased frequency and vivacity of dreaming and with such waking changes as loss of reality testing, visual hallucinations, and delusions. This suggested to Solms that structures in this region normally exercise some inhibitory function on the occurrence and intensity of dreaming. Finally, lesions in the dorsolateral prefrontal cortex, a region essential for executive functioning, goal-directed behavior, and self-monitoring, have no effect on dreaming, indicating that these functions are not significantly involved in the dreaming process.

An important strength of Solms' approach is that it links data concerning the brain directly to findings about dreams, rather than relying on indirect inferences based on more generic relationships between aspects of the brain and mental functioning. It should be noted that Solms' findings provide neurophysiological evidence for the crucial role of forebrain mechanisms in dreaming. Indeed, Solms goes farther than this. He reports that dreaming is preserved with lesions in the brain-stem area crucial for the generation of REM sleep, and he argues on this basis for an essential disconnection between REM sleep and dreaming. REM sleep, he postulates, is involved in dreaming only as one of many possible sources of activation. This has yet to be supported by sleep laboratory findings demonstrating that REM sleep is indeed absent in the patients with brain-stem lesions and preserved dreaming, and is perhaps the most speculative aspect of Solms' model. Solms integrates his findings closely with Freud's model of dreaming. The one major element of that model for which he feels there is not yet clear neurobiological evidence is that of censorship. $^{48}$

\section{Imaging Studies}

The development and application of brain imaging techniques is yielding another very significant new body of findings concerning the functioning of the brain during REM sleep. ${ }^{49-51}$ Although different groups have utilized different imaging techniques and subject procedures, striking consistencies have emerged. The groups of Maquet, Braun, and Nofzinger all report a very specific, selective pattern of activation of forebrain structures, suggesting that the brain is organized to carry out particular functions in a concerted fashion. Structures in the brain stem, thalamus, and basal forebrain that mediate arousal are activated. A major role for emotion and drive is suggested by high levels of activation of parts of the hypothalamus and the limbic and paralimbic systems; the amygdala may be particularly connected to the role of anxiety in dreaming. On the other hand, two of the groups, those of Maquet and Braun, found a widespread deactivation of the dorsolateral prefrontal cortex, which correlates well with the diminished executive functioning in dreams. Maquet et al, emphasizing the role of the amygdala, suggest that REM sleep is involved in processing "emotionally significant memories. ${ }^{, 49}$ Nofzinger and colleagues interpret the pattern of activation as supporting "the view that one function of REM sleep is the integration of neocortical activity with hypothalamic-basal forebrain regulatory and motivational reward mechanisms." They see their findings as consistent with a role for REM sleep in memory consolidation, and as "quite in accordance 
with older, more general views that REM sleep, and specifically dream content, is associated with internally generated, or instinctual behaviors that subserve adaptive mechanisms." 51

There is an impressively high degree of consistency between the imaging data and Solms' lesion findings, each thereby lending support to the other. ${ }^{44}$ Important aspects of a functional neuroanatomy of REM sleep, and of dreaming, have begun to be established. As technology develops and further data are collected, rapid advances in this area may be expected. Less certain is the degree to which these findings can be utilized to shed light on the understanding of dreams per se. The views of Hobson ${ }^{36}$ and $\mathrm{Kramer}^{52}$ may be compared for very different perspectives on this issue; the former essentially derives an entire understanding of dreaming from neurophysiology, the latter argues that neurophysiological findings at present provide no basis for understanding of the individual content or meaning of dreams and that efforts to utilize the findings in that way are highly reductionistic. Further, different authors may draw dramatically different inferences from essentially the same neurophysiological data. ${ }^{36,37}$ Clearly, caution must be exercised in attempts to correlate knowledge about the brain with dreaming. However, most would agree that at the least, the findings at present can lend support to some hypotheses regarding dreaming and make others seem less likely and may help to establish a framework in which a theory of dreaming must be cast.

\section{SOME RECENT THEORIES OF DREAMING}

Several of the more recent theories of dreaming have emphasized adaptive functions. One such model is that developed by Hartmann. ${ }^{53}$ His view is that the purpose of dreaming is to provide the dreamer with an opportunity to deal with their dominant emotional concern. The dream does this by utilizing an "autoassociative" mode in which connections are made more broadly and loosely than in directed waking thought, providing the dreamer with the opportunity to contextualize the dominant emotion in the form of an explanatory metaphor. This permits the dreamer both to calm and integrate their dominant emotional concern and enhances their capacity for future adaptations to similar emotional disturbances. Hartmann's theory derives in good part from his observations on the dreams of patients following exposure to trauma. Rather closely related functions for dreaming that incorporate problem-solving and affect regulation have been proposed by $\mathrm{Kramer}^{54}$ and Cartwright et al. ${ }^{55}$ An adaptive function also has a place in more recent psychoanalytic views. ${ }^{56}$

Another trend of thought in some recent work on dreaming is related to research on the involvement of REM sleep in learning. Most often, the type of learning cited is consolidation of procedural memory, ${ }^{24,57}$ or emotional learning. In research with rats, findings have been reported from unit cell recordings in the hippocampus to indicate that cells active during a learning task while awake are reactivated in a similar pattern during REM sleep; this has been seen as related to a process of memory consolidation. ${ }^{58}$ Maquet et al have reported analogous findings in humans using positron-emission tomography imaging; areas of the brain activated during training on a task were more active during subsequent REM sleep. ${ }^{59}$ Stickgold et al propose that in the context of a learning paradigm, "dreams represent the conscious awareness of complex brain systems involved in the reprocessing of emotions and memories during sleep." 60 They have presented novel data that show the frequent incorporation of imagery from a presleep learning task in sleep-onset hallucinations and speculatively extended these findings to propose that REM sleep dreaming utilizes memory in "an attempt to identify and evaluate novel cortical associations in the light of emotions." ${ }^{\text {60 }}$ Here, too, an adaptive function for dreaming, in some ways similar to that proposed by Hartmann, is evoked.

\section{CONCLUSION}

The survey above provides some idea of the vast array of sources and kinds of data that have been brought to bear in the effort to understand dreaming since the beginning of the laboratory era of sleep research. Dreams have been systematically collected in the laboratory under many different conditions, and experimental manipulations of dreams and of REM sleep have been performed. The relationship between the physiology of REM sleep and dreaming has been scrutinized. Recent rapid developments in the neurosciences have led to impressive advances in knowledge of the functioning of the brain during REM sleep and dreaming. At the same time, understanding of dreaming derived primarily from the intensive exploration of individual dreams in their subjective context retains an essential place. At this current point in the history of the field, there are widely differing views concerning how dreams should be seen in light of all the available data. We can look forward to continued stimulating growth in this field in the coming years.

\section{REFERENCES}

1. Aserinsky E, Kleitman N. Regularly occurring periods of eye motility, and concomitant phenomena, during sleep. Science 1953;118:273-274

2. Dement W, Kleitman N. Cyclic variations in EEG during sleep and their relation to eye movements, body motility, and dreaming. Electroencephalogr Clin Neurophysiol Suppl 1957;9:673-690

3. Foulkes D. Dream reports from different stages of sleep. J Abnorm Soc Psychol 1962;65:14-25 
4. Dement W, Kleitman N. The relation of eye movements during sleep to dream activity: an objective method for the study of dreaming. J Exp Psychol 1957;53:339-346

5. Snyder F. The phenomenology of dreaming. In: Madow L, Snow LH, eds. The Psychodynamic Implications of the Physiological Studies on Dreams. Springfield, IL: Charles C. Thomas; 1970:124-151

6. Goodenough DR. Dream recall: history and current status of the field. In: Arkin AM, Antrobus JS, Ellman SJ, eds. The Mind in Sleep: Psychology and Psychophysiology. Hillsdale, NJ: Lawrence Erlbaum Associates; 1978:113-140

7. Offenkrantz W, Rechtschaffen A. Clinical studies of sequential dreams. I. A patient in psychotherapy. Arch Gen Psychiatry 1963;8:497-508

8. Rechtschaffen A, Vogel G, Shaikun G. Interrelatedness of mental activity during sleep. Arch Gen Psychiatry 1963;9: 536-547

9. Roffwarg H, Dement W, Muzio J, et al. Dream imagery: relationship to rapid eye movements of sleep. Arch Gen Psychiatry 1962;7:235-258

10. Kohler WC, Coddington RD, Agnew HW. Sleep patterns in 2-year-old children. J Pediatr 1968;72:228-233

11. Foulkes D. Children's Dreaming and the Development of Consciousness. Cambridge, MA: Harvard University Press; 1999

12. Resnick J, Stickgold R, Rittenhouse CD, et al. Selfrepresentation and bizarreness in children's dream reports collected in the home setting. Conscious Cogn 1994;3:30-45

13. Salzarulo $P$, Chevalier A. Sleep problems in children and their relationship with early disturbances of the waking-sleeping rhythms. Sleep 1983;6:47-51

14. Dement W, Wolpert EA. The relation of eye movements, body motility, and external stimuli to dream content. J Exp Psychol 1958;55:543-553

15. Dement W. The effect of dream deprivation. Science 1960; 131:1705-1707

16. Dement W, Fisher C. Experimental interference with the sleep cycle. Can Psychiatr Assoc J 1963;8:400-405

17. Dement WC. Experimental dream studies. In: Masserman JH, ed. Science and Psychoanalysis. Vol. VII: Development and Research. New York: Grune \& Stratton; 1964:129162

18. Vogel GW. A review of REM sleep deprivation. Arch Gen Psychiatry 1975;32:749-761

19. Vogel GW, Thurmond A, Gibbons P, et al. REM sleep reduction effects on depressive syndromes. Arch Gen Psychiatry 1975;32:765-777

20. Dement W, Henry P, Cohen H, et al. Studies on the effect of REM deprivation in humans and in animals. In: Kety SS, Evarts EV, Williams HL, eds. Sleep and Altered States of Consciousness. Baltimore: Williams and Wilkins; 1967:456468

21. Dement W. The biological role of REM sleep (circa 1968). In: Kales A, ed. Sleep: Physiology and Pathology. Philadelphia: JB Lippincott; 1969:245-265

22. Steiner SS, Ellman SJ. Relation between REM sleep and intracranial self-stimulation. Science 1972;177:1122-1124

23. Smith CT. Sleep states and learning: a review of the animal literature. Neurosci Biobehav Rev 1985;9:157-168

24. Smith C. Sleep states and memory processes. Behav Brain Res 1995;69:137-145

25. Vertes RP, Eastman KE. The case against memory consolidation in REM sleep. Behav Brain Sci 2000;23:867-876
26. Fisher C, Byrne J, Edwards A, et al. A psychophysiological study of nightmares. J Am Psychoanal Assoc 1970;18:747782

27. Gastaut H, Broughton R. A clinical and polygraphic study of episodic phenomena during sleep. In: Wortis J, ed. Recent Advances in Biological Psychiatry. Vol. VII. New York: Plenum Press; 1965:197-221

28. Jacobson A, Kales A, Lehmann D, et al. Somnambulism: allnight electroencephalographic studies. Science 1965;148:975977

29. Schenck CH, Hurwitz TD, Mahowald MW. REM sleep behaviour disorder: an update on a series of 96 patients and a review of the world literature. J Sleep Res 1993;2:224231

30. Fisher C. Dreaming and sexuality. In: Loewenstein RM, Newman LM, Schur M, et al, eds. Psychoanalysis-A General Psychology: Essays in Honor of Heinz Hartmann. New York: International Universities Press; 1966:537569

31. Pivik RT. Tonic states and phasic events in relation to sleep mentation. In: Ellman SJ, Antrobus JS, eds. The Mind in Sleep: Psychology and Psychophysiology. 2nd ed. New York: John Wiley \& Sons; 1991:214-247

32. Molinari S, Foulkes D. Tonic and phasic events during sleep: psychological correlates and implications. Percept Mot Skills 1969;29:343-368

33. Freud S. The interpretation of dreams. In: Strachey J, trans. and ed. The Standard Edition of the Complete Psychological Works of Sigmund Freud. Vols. IV and V. London, UK: The Hogarth Press; 1953(orig. published 1900)

34. Vogel GW. Critique of current dream theories. Behav Brain Sci 2000;23:1014-1016

35. Shevrin H, Fisher C. Changes in the effects of a waking subliminal stimulus as a function of dreaming and nondreaming sleep. J Abnorm Psychol 1967;72:362-368

36. Hobson JA. The new neuropsychology of sleep: implications for psychoanalysis. Neuro-Psychoanalysis 1999;1:157-183

37. Solms M. Commentary on the new neuropsychology of sleep. Neuro-Psychoanalysis 1999;1:183-195

38. Fisher C. Psychoanalytic implications of recent research on sleep and dreaming. Part I: empirical findings. Part II: implications for psychoanalytic theory. J Am Psychoanal Assoc 1965;13:271-303

39. McCarley RW, Hobson JA. Neuronal excitability modulation over the sleep cycle: a structural and mathematical model. Science 1975;189:58-60

40. Hobson JA, McCarley RW. The brain as a dream state generator: an activation-synthesis hypothesis of the dream process. Am J Psychiatry 1977;134:1335-1348

41. Vogel GW. An alternative view of the neurobiology of dreaming. Am J Psychiatry 1978;135:1531-1535

42. Rechtschaffen A. The single-mindedness and isolation of dreams. Sleep 1978;1:97-109

43. Jones BE. The interpretation of physiology. Behav Brain Sci 2000;23:955-956

44. Hobson JA, Pace-Schott EF, Stickgold R. Dreaming and the brain: toward a cognitive neuroscience of conscious states. Behav Brain Sci 2000;23:793-842

45. Solms M. New findings on the neurological organization of dreaming: implications for psychoanalysis. Psychoanal Q 1995;64:43-67

46. Solms M. The Neuropsychology of Dreams. Mahwah, NJ: Lawrence Erlbaum Associates; 1997 
47. Solms M. Dreaming and REM sleep are controlled by different brain mechanisms. Behav Brain Sci 2000;23:843850

48. Solms M. Ongoing discussion. Commentary on J. Allan Hobson and Edward Pace-Schott's response. Neuro-Psychoanalysis 2000;2:193-201

49. Maquet P, Peters J-M, Aerts J, et al. Functional neuroanatomy of human rapid-eye-movement sleep and dreaming. Nature 1996;383:163-166

50. Braun AR, Balkin TJ, Wesensten NJ, et al. Regional cerebral blood flow throughout the sleep-wake cycle. An $\mathrm{H}_{2}{ }^{15} \mathrm{O}$ PET study. Brain 1997;120:1173-1197

51. Nofzinger EA, Mintun MA, Wiseman MB, et al. Forebrain activation in REM sleep: an FDG PET study. Brain Res 1997;770:192-201

52. Kramer M. Dreaming has content and meaning not just form. Behav Brain Sci 2000;23:959-961

53. Hartmann E. Outline for a theory on the nature and functions of dreaming. Dreaming 1996;6:147-170

54. Kramer M. The selective mood regulatory function of dreaming: an update and revision. In: Moffitt A, Kramer
M, Hoffmann R, eds. The Functions of Dreaming. Albany, NY: State University of New York Press; 1993: 139-195

55. Cartwright R, Luten A, Young M, et al. Role of REM sleep and dream affect in overnight mood regulation: a study of normal volunteers. Psychiatry Res 1998;81:1-8

56. Altman L. Adaptive ego and superego in the dream. In: Altman L. The Dream in Psychoanalysis. Revised ed. New York: International Universities Press Inc.; 1975:231252

57. Smith C. Sleep states, memory processes and synaptic plasticity. Behav Brain Res 1996;78:49-56

58. Pavlides C, Winson J. Influences of hippocampal place cell firing in the awake state on the activity of these cells during subsequent sleep episodes. J Neurosci 1989;9:2907-2918

59. Maquet P, Laureys S, Peigneux $\mathrm{P}$, et al. Experiencedependent changes in cerebral activation during human REM sleep. Nat Neurosci 2000;3:831-836

60. Stickgold R, Hobson JA, Fosse R, et al. Sleep, learning, and dreams: off-line memory reprocessing. Science 2001;294: 1052-1057 